\title{
Mesure de la production laitière de brebis Hamra durant les phases d'allaitement et de traite
}

\author{
MT Benyoucef 1, A Ayachi 2 \\ 1 INA, Département des productions animales, 16200 El Harrach, Alger; \\ 2 Ferme expérimentale ITEBO, 20100 Ain-EI-Hadjar, Saïda, Algérie
}

(Reçu le 7 septembre 1990; accepté le 13 novembre 1990)

\begin{abstract}
Résumé - La production laitière de 27 brebis Hamra âgées de 4 ans a été étudiée, ainsi que son influence sur la croissance des agneaux. Durant la période d'allaitement, la production est estimée à $70 \mathrm{~kg}$ de lait pour les brebis allaitant des jumeaux (B) et à $56 \mathrm{~kg}$ pour les brebis allaitant des agneaux uniques $(A)$.

Après $42 \mathrm{j}$ d'allaitement la production laitière à la traite est la même pour les groupes $A$ et $B$ (53 et $54 \mathrm{~kg}$ ).

II existe une corrélation étroite entre la production laitière estimée en période d'allaitement et le gain de poids pendant cette période : elle est maximale au cours des 3 ou 4 premières semaines $(r=0,83$, groupe A et $r=0,74$ groupe B). L'indice de consommation est de 5,8 pour les agneaux doubles et de 7,0-7,3 pour les simples, soit 6,5 pour l'ensemble des agneaux.
\end{abstract}

production laitière / croissance / agneau / brebis Hamra

Summary - Measure of milk yield of Hamra ewes during the suckling and milking periods. The milk yield of 27 Hamra ewes of 4 yr age was studied, also its influence on the growth of their lambs.

During the suckling period, the milk yield was estimated at $70 \mathrm{~kg}$ and $55-57 \mathrm{~kg}$ for the twin and singlesuckling ewes respectively.

However, during the milking period, the milk yield was the same for the differents groups ( $A: 53 \mathrm{~kg}$ and $B: 54 \mathrm{~kg}$ ).

There is a positive correlation between the milk suckled and the growth of lambs for all the suckling period (42 d). This correlation is stronger during the first $28 \mathrm{~d}$ of suckling $(\mathrm{A}, \mathrm{r}=0.83$ and $\mathrm{B}, \mathrm{r}=$ 0.74 ). The feed efficiency is 5.8 and 7.0-7.3 for twin and single lambs respectively, ie 6.5 on average.

milk yied / growth / lamb / Hamra ewe

\section{INTRODUCTION}

La production de lait de brebis et des produits dérivés est trés développée dans les pays méditerranéens (Boyazoglu, 1963, 1988). Selon le rapport de la Fédération Internationale des laiteries (FIL) en 1983, la consommation de lait ovin cru est rare, mais celle des produits fermentés (yaourt) et des fromages (Ricotta, Manchego, Rocquefort, etc.) est importante.

En Algérie, la production de lait de brebis en vue d'une consommation humaine dépend essentiellement de l'année (pluviométrie) et par conséquent de l'état des pâturages naturels (parcours steppiques). Lorsque l'année est favorable, les brebis sont traites 1 fois avant la tétée des 
agneaux durant l'allaitement, et 2 fois aprés le sevrage des agneaux (2-4 mois).

En année moyenne, les brebis traites sont celles ayant perdu tôt leurs agneaux (mortalité périnatale, agneau adopté etc.) ou dont les agneaux sont sevrés. En année défavorable, la traite est pratiquement impossible : le problème se pose en termes de survie aussi bien de la mère que de son agneau.

La race ovine Hamra (2 500000 têtes) est élevée en Algérie, surtout dans la steppe occidentale ou elle est dénommée également Daghma, ce qui signifie, "mouton à tête marron roussâtre". Les brebis pèsent en moyenne $40 \mathrm{~kg}$.

L'âge à la puberté est de 6-8 mois, le taux de fertilité de $90-95 \%$ et la taille de portée 1,1-1,2 (EROPA, 1980).

L'objet de l'étude est d'évaluer le niveau de production laitière d'un troupeau de brebis de cette race dans une ferme expérimentale située en zone céréalière pendant la campagne 1988-1989.

\section{MATÉRIEL ET MÉTHODES}

L'expérimentation se déroule du 23 octobre 1988 au 24 février 1989, à la ferme expérimentale de Ain El Hadjar qui se situe dans une zone céréalière comprise entre les isohyètes 400 et $500 \mathrm{~mm}$.

Le troupeau se compose de 27 brebis Hamra âgées de 4 ans et réparties en 3 groupes de 9 selon le sexe et le nombre d'agneaux allaités. Les brebis du groupe $A_{1}$ allaitent des mâles simples, celles du groupe $A_{2}$ des femelles simples et celles du groupe $B$ des jumeaux.

Les brebis reçoivent une ration de base de foin de vesce-avoine avec un complément de concentré dosant 0,79 UF et $130 \mathrm{~g} . \mathrm{MAD} / \mathrm{kg}$ et distribué à raison de $0,6 \mathrm{kg.j} \mathrm{j}^{-1}$ par mère en fin de gestation et durant la lactation.

Durant les 6 semaines d'allaitement, les brebis sont logées individuellement avec leurs pro- duits et sont contrôlées chaque semaine. La production laitière est estimée par la méthode hormonale propsée par Mc Cance (1959) et révisée par Doney et al (1979). Le jour du contrôle, les brebis sont séparées de leurs produits à $9 \mathrm{~h}$ et soumises à une traite d'épuisement. Quatre heures après, c'est-à-dire à $13 \mathrm{~h}$, une deuxième traite est réalisée par injection intra-jugulaire de $5 \mathrm{UI}$ d'ocytocine réparties en 3 doses (2-2-1). La quantité de lait obtenue à la deuxième traite d'épuisement est multipliée par 6 pour donner une estimation de la quantité de lait sécrétée en $24 \mathrm{~h}$.

Durant la période de traite, les brebis sont traites matin et soir. Les contrôles laitiers sont réalisés tous les $15 \mathrm{j}$, le dernier contrôle ayant lieu lorsque le niveau de production moyen du troupeau devient $<300 \mathrm{~g} / \mathrm{j}$. L'estimation de la quantité de lait totale produite est faite par la méthode Fleischmann (Prud'Hon, 1983).

Les agneaux sont pesés tous les $21 \mathrm{j}$, de façon à calculer leurs poids à des âges types. Pour les 3 groupes, on a calculé leur indice de consommation, c'est-à-dire le rapport entre la quantité de lait consommé et le gain de poids vif pendant la même période.

\section{RÉSULTATS}

\section{Quantité de lait}

La figure 1 représente la production moyenne de chacun des 3 groupes, ainsi que la moyenne du troupeau expérimental. Des différences importantes de production laitière sont observées durant les $42 \mathrm{j}$ d'allaitement selon le nombre d'agneaux allaités. Les brebis allaitant des doubles (groupe B) produisent beaucoup plus de lait que celles ayant des simples (groupe A). La différence est statistiquement très significative pour les 4 premiers contrôles et significative pour les 2 contrôles suivants. Le maximum de production est obtenu à $14 \mathrm{j}$ de lactation chez les brebis allaitant 2 agneaux $(2,13 \mathrm{~kg} / \mathrm{j})$ et à $21 \mathrm{j}$ chez les brebis allaitant 1 agneau $(1,55 \mathrm{~kg} / \mathrm{j})$. Après le maximum de production et 


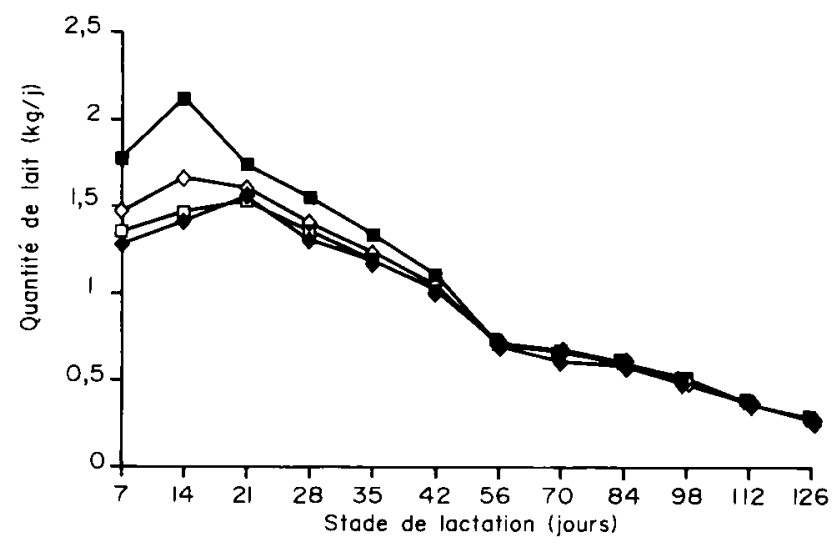

Fig 1. Quantité de lait produite en phase d'allaitement et de traite.

doubles; $\square$ simples $\sigma$; : simples $Q ; \diamond: C$ moyenne.

jusqu'au dernier contrôle en période d'allaitement, la baisse de production est de l'ordre de $10,4 \%$ dans le groupe $B$ et de $8,3 \%$ dans le groupe $A ;$ de sorte qu'en fin d'allaitement, la production est respectivement de 1,02 et $1,11 \mathrm{~kg} / \mathrm{j}$ dans les groupes A et $B$.

Au cours des 42 j d'allaitement, les brebis allaitant des doubles ont une production supérieure de 23 et $27 \%$ à celles allaitant des simples $(70 \mathrm{~kg}$ vs 55 et $57 \mathrm{~kg}$ ), soit une production moyenne journalière de $1,67 \mathrm{~kg}$ et $1,31 \mathrm{~kg}$ et $1,36 \mathrm{~kg}$ (tableau I).
Pendant la période de traite, l'évolution de la production laitière est comparable dans les groupes $A$ et $B$, avec cependant une légère supériorité pour les brebis ayant allaité 2 agneaux durant les jours qui suivent le sevrage.

La chute de production au sevrage (rapport entre la production au premier contrôle post-sevrage et celle du dernier contrôle d'allaitement) est en moyenne de $37 \%$ pour les brebis avec 2 agneaux contre 30 et $32 \%$ pour les brebis avec 1 agneau.

Tableau I. Production laitière pendant l'allaitement et la traite.

\begin{tabular}{lccc}
\hline & $\begin{array}{c}\text { Brebis allaitant } \\
\text { 1 agneau mâle }\end{array}$ & $\begin{array}{c}\text { Brebis allaitant } \\
\text { 1 agneau femelle }\end{array}$ & $\begin{array}{c}\text { Brebis allaitant } \\
\text { 2 agneaux }\end{array}$ \\
\hline Nbre de brebis & 09 & 09 & 09 \\
42 j d'allaitement $(\mathrm{kg})$ & $57 \pm 4$ & $55 \pm 9$ & $70 \pm 9$ \\
Moyenne par jour (kg/j) & 1,36 & 1,31 & 1,67 \\
98 j de traite (kg) & $54 \pm 4$ & $53 \pm 7$ & $54 \pm 5$ \\
Moyenne par jour (kg/j) & 0,55 & 0,54 & 0,55 \\
Poids vif $(\mathrm{kg})$ & $42,3 \pm 3,1$ & $41,1 \pm 2,8$ & $43,5 \pm 3,7$ \\
\hline
\end{tabular}


Durant la période de traite, la quantité de lait produite est de $54 \mathrm{~kg}$ pour les brebis du groupe B contre $53 \mathrm{~kg}$ pour celles du groupe $A$, soit respectivement une production moyenne journalière de 0,55 et $0,54 \mathrm{~kg}$ (tableau I). Par ailleurs, quelle que soit la période considérée (allaitement ou traite) il n'existe aucune différence entre les productions des brebis ayant allaité un mâle ou une femelle $\left(A_{1}\right.$ et $\left.A_{2}\right)$.

\section{Croissance des agneaux}

L'évolution du poids des agneaux, de la naissance à l'âge de 120 j, est présentée à la figure 2. Pendant la période d'allaitement, la croissance moyenne journalière est de $186 \mathrm{~g}$ pour les mâles simples, de
$177 \mathrm{~g}$ pour les femelles simples et de $145 \mathrm{~g}$ pour les doubles. Après sevrage, les agneaux simples (A1 et $A 2$ ) ont une croissance significativement supérieure à celle des doubles (groupe B), soit 138 et $156 \mathrm{~g}$. Entre sexes, la différence est significative en faveur des mâles simples.

\section{Relation entre le gain de poids des agneaux et la production laitière estimée de leur mère, en période d'allaitement}

Le tableau II montre qu'il existe une relation étroite entre les 2 critères : la corrélation maximale est atteinte à la $3^{e}$ semaine chez les doubles $(0,74)$ et à la $4^{e}$ semaine chez les simples $(0,83)$.

Tableau II. Coefficient de corrélation entre le gain de poids des agneaux allaités et la production de leur mère.

\begin{tabular}{lcccccc} 
& \multicolumn{7}{c}{ Période d'allaitement (j) } \\
& $0-7 j$ & $0-14 j$ & $0-21 j$ & $0-28 j$ & $0-35 j$ & $0-42 j$ \\
\hline Simples & 0,24 & 0,50 & 0,62 & 0,83 & 0,51 & 0,39 \\
Doubles & 0,54 & 0,56 & 0,74 & 0,67 & 0,47 & 0,27 \\
\hline
\end{tabular}

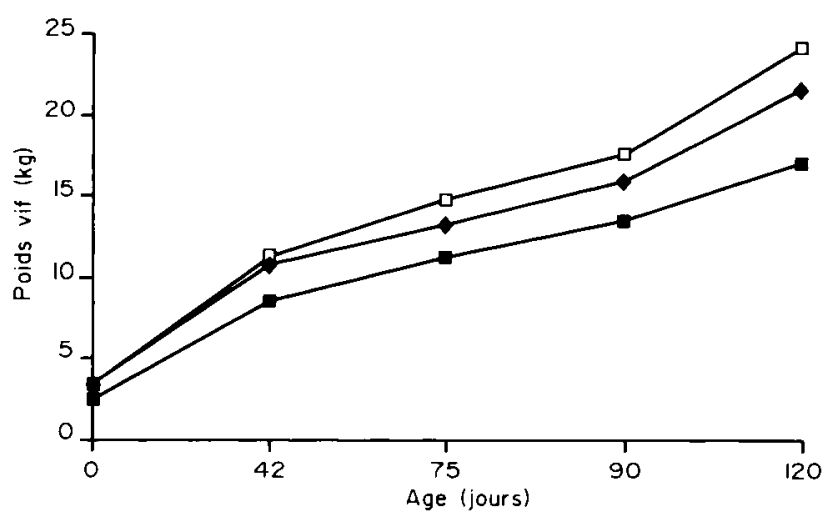

Fig 2. Évolution du poids vif des agneaux.

$\rightarrow$ doubles; $\square: \sigma^{\prime \prime}$ simples; - : $९$ simples 
En ce qui concerne l'indice de consommation, il est de 5,8 pour les doubles et de 7,0 et 7,3 pour les simples, mais les différences ne sont pas significatives; l'indice moyen des 36 agneaux est de 6,5.

\section{DISCUSSION}

En période d'allaitement, les brebis allaitant des doubles ont donc une production laitière supérieure (23-27\%) à celles allaitant des simples, par suite d'une plus grande stimulation de la mamelle. Cette supériorité correspond aux résultats de beaucoup d'auteurs, notamment Ricordeau et Denamur (1962) sur la race Préalpes du Sud et Katsaounis et Zygouiannis (1984) sur la race Karagouniko dont les estimations sont respectivement de $37 \%$ et $42 \%$.

L'importance de la perte de production constatée au moment de sevrage $(+30 \%)$ et la disparition de l'effet favorable du nombre d'agneaux allaités sur la production laitière à la traite s'expliquent par la différence de fréquence de vidange du pis entre l'allaitement (plusieurs tétées par jour) et la traite (2 fois par jour), comme cela a été vérifié par Labussière et al (1974 et 1978). La majorité des auteurs (Ricordeau et Denamur, 1962; Mavrogenis, 1982; Hadjipanayiotou et Economides,
1986 et Barillet, 1989) trouvent que l'effet de l'allaitement double sur la production laitière traite disparaît aprés le sevrage, alors que Katsaounis et Zygouiannis (1984) observent encore un effet favorable en race Karagouniko.

En période de traite, les performances des brebis Hamra, (53,5 kg en 98 j de traite) sont inférieures à celles des Lacaune (France) ou Sarde (Italie), qui font l'objet de programmes de sélection; mais elles sont comparables à celles des brebis Karaman rouge en Turquie ou Sicilo-Sarde en Tunisie (tableau III). Ces comparaisons entre performances brutes n'ont cependant qu'une valeur indicative, compte tenu du poids des brebis, du niveau d'alimentation et de la diversité des systèmes d'élevage.

La corrélation entre le gain de poids des agneaux et la production laitière des mères est maximale à 28 j pour les simples et à 21 j pour les doubles, comme si ceux-ci étaient capables de consommer plus tôt de l'aliment solide. Dans l'ensemble, cependant, nos observations correspondent à celles obtenues avec les races Préalpes du Sud par Ricordeau et Boccard (1961), Blackface par Peart (1967) et Barbarine par Khaldi (1983).

L'indice de consommation des agneaux Hamra $(6,5)$ est supérieur à celui observé par Ricordeau et Boccard (1961) en race Préalpes du Sud $(5,0)$ et Economides

Tableau III. Production laitière à la traite de quelques races de brebis méditerranéennes.

\begin{tabular}{lcccl}
\hline $\begin{array}{c}\text { Type } \\
\text { génétique }\end{array}$ & $\begin{array}{c}\text { Production laitière } \\
\text { à la traite }\end{array}$ & $\begin{array}{c}\text { Durée de traite } \\
(\mathrm{j})\end{array}$ & $\begin{array}{c}\text { Poids vif } \\
(\mathrm{kg})\end{array}$ & \multicolumn{1}{c}{ Sources } \\
\hline & $211(\mathrm{~h})$ & 165 & - & Barillet, 1989 \\
Lacaune & $178(\mathrm{~h})$ & 180 & 42 & FlL, 1983 \\
Sarde & $45(\mathrm{~kg})$ & 140 & 45 & Hatziminaglou et al, 1987 \\
Karaman rouge & $60(\mathrm{~kg})$ & - & 45 & EROPA, 1980 \\
Sicilo-Sarde & & & & \\
\hline
\end{tabular}


(1984) en race Chios $(4,9$ à 5,2) mais il est comparable aux indices de Neiding et Iddings (1919) en race Lincoln $(6,5)$, Barnicoat et al (1949) en race Romey-Marsh $(7,4)$ et Amegee (1984) en race Vogan $(6,4) \cdot x$

\section{CONCLUSION}

A notre connaissance, l'évaluation de la production laitière des brebis algériennes, et notamment de la race Hamra, n'a jamais été réalisée. Cette étude limitée à des brebis Hamra, apporte donc une contribution originale sur les aptitudes de cette race. Elle montre également l'intérêt et la possibilité de développer la pratique de la traite de brebis en zone céréalière et d'instaurer un contrôle laitier pendant la période de traite. Ceci suppose cependant l'implication d'un grand nombre d'éleveurs motivés dans un programme de contrôle laitier à mettre en place aussi bien dans les fermes expérimentales que les élevages privés. Ce contrôle doit estimer la production de lait à la traite après une durée d'allaitement bien définie (par exemple $42 \mathrm{j}$ ), mais aussi tenir compte des facteurs de variation tels que l'année, la période de mise bas, le troupeau, le rang de lactation etc.

Dans une perspective de développement de la production de lait de brebis en Algérie, il serait utile de comparer les races locales, mais surtout de mettre en place un schéma de sélection pour exploiter la variabilité intraraciale. Ceci suppose le contrôle des saillies, l'utilisation de l'insémination artificielle et la pratique des accouplements raisonnés entre les meilleurs béliers et les meilleures brebis.

\section{REMERCIEMENTS}

Les auteurs remercient $G$ Ricordeau et $F$ Barillet (INRA - SAGA - Toulouse).

\section{REFERENCES}

Amégée $Y$ (1984) Le mouton de Vogan (croisé Djallonké $x$ Sahélien) au Togo. 1. La production lactée et ses relations avec la croissance des agneaux. Rev Med Pays Trop 37, 82-90

Barillet $F$ (1989) Expression de la production laitière à la traite des brebis lacaune en système allaitement $x$ traite mécanique. $4 \mathrm{e}$ symp International sur la traite mécanique des petits ruminants. Tel-Ariv, Israël, 13-19 Septembre 1989

Barnicoat CR, Logan AG, Grant Al (1949) Milk secretion studies with the New Zeland Romey ewes. J Agric Sci 39, 44-55 and 237248

Boyazoglu JG (1963) Aspects quantitatifs de la production laitière des brebis 1 . Mise au point bibliographique. Ann Zootech 12, 237-296

Boyazoglu JG (1988) La production laitière ovine en systèmes extensifs méditérranéens. In : Le lait dans la région méditérranéenne Symp Rabat, 25-27 Oct $18 \mathrm{p}$

Doney JM, Peart JN, Smith WF, Louda F (1979) A consideration of the techniques for estimation of milk yield by suckled sheep and a comparison of estimates obtained by two methods in relation to the effect of breed, level of production and stage of lactation. $J$ Agric Sci (Camb) 92, 123-132

Economides S (1984) the effects of lamb - Weaning age on the milk yield of Chios ewes and the growth and carcass gain of lambs. Tech Bull Agric Res Inst Nicosia 59, 7p

Encyclopédie des races ovines des pays arabes (1980) $T_{1}$ OAECCIA

EROPA (1980) Organisation arabe pour le développement de l'agriculture (encyclopédie éditée en arabe) 
FIL (1983) Production et utilisation de lait de brebis et de chèvre Doc $n^{\circ} 158$

Hadjipanayiotou MJ, Economides S (1986) The effect of rearing type on the pre-and post wearing lactation performance of Chios sheep and the growth rate of the lambs. Tech Bull Agric Res Inst Nicosia 78, 7p

Hatziminaglou J, Alifakiotis R, Zervas N (1987) Wool, meat and milk yield from the carpet wool producing sheep breeds in the mediterraneen region. $38^{e}$ reunion de la FEZ, Lisbonne (1987)

Katsaounis N, Zygouiannis D (1984) Production laitière et composition du lait de brebis de race Karagouniko. 1. Brebis allaitant des agneaux de race Karagouniko. Rec Med Vet $160,745-753$

Khaldi G (1983) Influence du niveau alimentaire en fin de gestation et pendant la lactation sur la production laitière des brebis et la croissance des agneaux de race Barbarine en année sèche. Ann INRAT 3, 1-32

Labussière J, Combaud JF, Petrequin P (1974) Influence de la fréquence des traites et des tétées sur la production laitière des brebis Préalpes du Sud. Ann Zootech 23, 445-457

Labussière J, Combaud JF, Petrequin p (1978) Influence respective de la fréquence quotidienne des évacuations mammaires et des stimulations du pis sur l'entretien de la sécrétion lactée chez la brebis. Ann Zootech 27, 127-137

Mc Cance $J(1959)$ The determination of milk yield in the Merino ewes. Aust $J$ Agric Res 10, 839-853

Mavrogenis AP (1982) Environmental and genetic factors influencing milk production and lamb output of Chios sheep. Livest Prod Sci 8, 519-527

Neiding RE, Iddings, EJ (1919) Quantity and composition of ewe's milk: its relations to the growth of lambs. J Agric Res 17, 19-3

Peart JN (1967) The effects of differents levels of nutrition during late pregnancy on the subsequent milk production of Blackface ewes and on the growth of their lambs. J Agric Sci $68,365-371$

Prud'Hon $M$ (1983) Amélioration génétique de la production laitière bovine. Cours Polycopie ENSA Montpellier

Ricordeau G, Boccard R (1961) Relation entre la quantité de lait consommé par les agneaux et leur croissance. Ann Zootech 10, 113-125

Ricordeau G, Denamur R (1962) Production laitière des brebis Préalpes du Sud pendant les phases d'allaitement, de sevrage et de traite. Ann Zootech 11, 5-38 